\title{
Phase Control of Spontaneous Emission
}

\author{
E. Paspalakis and P. L. Knight \\ Optics Section, Blackett Laboratory, Imperial College, London SW7 2BZ, United Kingdom
}

(Received 17 November 1997)

\begin{abstract}
We use the phase difference of two lasers with equal frequencies for the control of spontaneous emission in a four-level system. Effects such as extreme spectral narrowing and selective and total cancellation of fluorescence decay are shown as the relative phase is varied. [S0031-9007(98)06540-5]
\end{abstract}

PACS numbers: $32.80 . \mathrm{Qk}, 32.50 .+\mathrm{d}$

The control of spontaneous emission has attracted much attention for many years. For atoms in free space, atomic coherence and quantum interference are the basic phenomena for controlling spontaneous emission [13]; these have potential applications to lasing without inversion [4-10]. Zhu, Scully, and co-workers have studied the quenching of spontaneous emission using an open V-type atom [11], and gave an experimental verification of their predictions [12].

Here, we study the potential for coherent control in a driven quantum system, using the relative phase between two lasers with equal frequencies $\omega_{a}=\omega_{b}=\omega$ which couple the ground state with the two excited states (see Fig. 1). These laser fields may be distinguished by their different transition characteristics [13,14]. In this way we can obtain efficient control, spectral narrowing, and quenching of spontaneous emission even if we have nontrapping conditions that do not allow control when a single laser is used. The use of two lasers makes the system independent of restrictions involving matrix elements to satisfy the trapping condition of Ref. [11]. Phase dependent effects in spontaneous emission spectra were recently studied in a $\Lambda$-type atom [15] and for an atom near the edge of a photonic band gap [16]. The effects of strong bichromatic excitation in the fluorescence spectrum from a two-level atom have also been studied $[17,18]$.

We use here the wave function approach, and assume that the atom is excited to a superposition of states $|0\rangle$, $|1\rangle,|2\rangle$. We apply the Weisskopf-Wigner theory $[2,19]$ and obtain the resulting equations for the probability amplitudes $(\hbar=1)$,

$$
\begin{aligned}
i \dot{c}_{0}(t)= & \Omega_{01} c_{1}(t)+\Omega_{02} c_{2}(t), \\
i \dot{c}_{1}(t)= & \Omega_{10} c_{0}(t)+\left(\delta_{1}-i \frac{\Gamma_{1}}{2}\right) c_{1}(t) \\
& -i p \frac{\sqrt{\Gamma_{1} \Gamma_{2}}}{2} c_{2}(t), \\
i \dot{c}_{2}(t)= & \Omega_{20} c_{0}(t)-i p \frac{\sqrt{\Gamma_{1} \Gamma_{2}}}{2} c_{1}(t) \\
& +\left(\delta_{2}-i \frac{\Gamma_{2}}{2}\right) c_{2}(t),
\end{aligned}
$$

$$
i \dot{c}_{\mathbf{k}}(t)=\delta_{\mathbf{k}} c_{\mathbf{k}}(t)-i g_{\mathbf{k} 1} c_{1}(t)-i g_{\mathbf{k} 2} c_{2}(t) .
$$

Here, $\Omega_{0 m}=\Omega_{m 0}^{*}=\Omega_{0 m}^{a} e^{i \delta \phi}+\Omega_{0 m}^{b}$ with $\Omega_{n m}^{l}$ the Rabi frequency for the $|n\rangle \rightarrow|m\rangle$ transition due to laser $l$, which we assume to be real and $\delta \phi=\phi_{a}-\phi_{b}$ is the phase difference of the two lasers, which is used to control the system. Also, $\delta_{m}=\omega_{m}-\omega_{0}-\omega$ is the detuning from state $|m\rangle(m=1,2)$ where the radiative shifts have been omitted, $\delta_{\mathbf{k}}=\omega_{\mathbf{k}}-\omega+\omega_{3}-\omega_{0}$, and $\Gamma_{m}=$ $2 \pi\left|g_{\mathbf{k} m}\right|^{2} D\left(\omega_{m 3}\right)$ is the spontaneous decay rate of state $|m\rangle(m=1,2)$, where $\mathbf{k}$ denotes both the momentum vector and the polarization of the emitted photon. $D\left(\omega_{m 3}\right)$ denotes the mode density at frequency $\omega_{m 3}(m=1,2) ; p$ denotes the alignment of the two dipole moment matrix elements $\vec{\mu}_{n m}\left(p \equiv \vec{\mu}_{13} \cdot \vec{\mu}_{32} /\left|\vec{\mu}_{13}\right|\left|\vec{\mu}_{32}\right|\right)$, and plays an important role in spontaneous emission cancellation [11].

For the (long time) spontaneous emission spectrum $S\left(\delta_{\mathbf{k}}\right)$ we calculate $c_{\mathbf{k}}(t \rightarrow \infty)$, as $S\left(\delta_{\mathbf{k}}\right)=\Gamma_{m} \mid c_{\mathbf{k}}(t \rightarrow$ $\infty)\left.\right|^{2} / 2 \pi\left|g_{\mathbf{k} m}\right|^{2}(m=1,2)$. We use the Laplace transform method [19] and the final value theorem to obtain

$$
c_{\mathbf{k}}(t \rightarrow \infty)=\frac{-g_{\mathbf{k} 1} K\left(\delta_{\mathbf{k}}\right)-g_{\mathbf{k} 2} L\left(\delta_{\mathbf{k}}\right)}{D\left(\delta_{\mathbf{k}}\right)},
$$

where

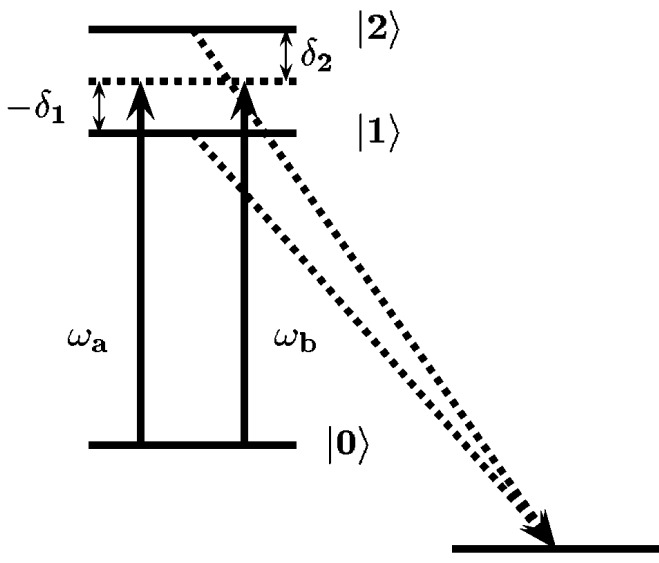

FIG. 1. The system under consideration. The ground state $|0\rangle$ is coupled to the excited states $|1\rangle,|2\rangle$ by two lasers of equal frequencies. The excited states decay solely to a common state $|3\rangle$. 


$$
\begin{aligned}
K\left(\delta_{\mathbf{k}}\right)= & i c_{0}(0)\left[\Omega_{10}\left(\delta_{\mathbf{k}}-\delta_{2}+i \frac{\Gamma_{2}}{2}\right)-i \Omega_{20} p \frac{\sqrt{\Gamma_{1} \Gamma_{2}}}{2}\right]+i c_{1}(0)\left[\delta_{\mathbf{k}}\left(\delta_{\mathbf{k}}-\delta_{2}+i \frac{\Gamma_{2}}{2}\right)-\left|\Omega_{02}\right|^{2}\right] \\
& -i c_{2}(0)\left[i \delta_{\mathbf{k}} p \frac{\sqrt{\Gamma_{1} \Gamma_{2}}}{2}-\Omega_{10} \Omega_{02}\right], \\
L\left(\delta_{\mathbf{k}}\right)= & i c_{0}(0)\left[\Omega_{20}\left(\delta_{\mathbf{k}}-\delta_{1}+i \frac{\Gamma_{1}}{2}\right)-i \Omega_{10} p \frac{\sqrt{\Gamma_{1} \Gamma_{2}}}{2}\right]-i c_{1}(0)\left[i \delta_{\mathbf{k}} p \frac{\sqrt{\Gamma_{1} \Gamma_{2}}}{2}-\Omega_{01} \Omega_{20}\right] \\
+ & i c_{2}(0)\left[\delta_{\mathbf{k}}\left(\delta_{\mathbf{k}}-\delta_{1}+i \frac{\Gamma_{1}}{2}\right)-\left|\Omega_{01}\right|^{2}\right], \\
D\left(\delta_{\mathbf{k}}\right)= & \delta_{\mathbf{k}}\left[\left(\delta_{\mathbf{k}}-\delta_{1}+i \frac{\Gamma_{1}}{2}\right)\left(\delta_{\mathbf{k}}-\delta_{2}+i \frac{\Gamma_{2}}{2}\right)+p^{2} \Gamma_{1} \Gamma_{2} / 4\right] \\
& -\left[\left|\Omega_{01}\right|^{2}\left(\delta_{\mathbf{k}}-\delta_{2}+i \frac{\Gamma_{2}}{2}\right)+\left|\Omega_{02}\right|^{2}\left(\delta_{\mathbf{k}}-\delta_{1}+i \frac{\Gamma_{1}}{2}\right)\right]+i p \frac{\sqrt{\Gamma_{1} \Gamma_{2}}}{2}\left[\Omega_{10} \Omega_{02}+\Omega_{01} \Omega_{20}\right] .
\end{aligned}
$$

Initially, we suppose laser $\omega_{a}$ drives only the $|0\rangle \leftrightarrow$ $|1\rangle$ transition and laser $\omega_{b}$ drives only the $|0\rangle \leftrightarrow|2\rangle$ transition, so that $\Omega_{01}^{b}=\Omega_{02}^{a}=0$. We are interested in conditions that will trap population in the system. The usual approach is to diagonalize the Hamiltonian of Eqs. (1)-(3) and search for positive (or zero) solutions of its characteristic equation $[D(\lambda)=0]$. There are two distinct conditions for population trapping. The first gives a zero root to the characteristic equation and occurs if

$$
\begin{gathered}
\delta_{1}\left(\Omega_{02}^{b}\right)^{2}+\delta_{2}\left(\Omega_{01}^{a}\right)^{2}=0, \\
\Gamma_{2}\left(\Omega_{01}^{a}\right)^{2}+\Gamma_{1}\left(\Omega_{02}^{b}\right)^{2}-2 p \Gamma_{1} \Gamma_{2} \Omega_{01}^{a} \Omega_{02}^{b} \cos [\delta \phi]=0 .
\end{gathered}
$$

These are obtained by setting the constant part of the characteristic equation to zero. From Eq. (10) we obtain

$$
p \cos [\delta \phi]= \pm 1, \quad \sqrt{\Gamma_{2}} \Omega_{01}^{a}= \pm \sqrt{\Gamma_{1}} \Omega_{02}^{b} .
$$

The second part of Eq. (11) can be satisfied by appropriately choosing the laser intensities such that $\sqrt{I_{a}} / \sqrt{I_{b}}=$ $\pm \sqrt{\Gamma_{1}} A_{02} / \sqrt{\Gamma_{2}} A_{01}$, as $\Omega_{n m}^{l} \equiv A_{n m} \sqrt{I_{l}}$, where $I_{l}$ is the intensity of laser $\omega_{l}$ (or its generalization for a multiphoton transition). The first part of Eq. (11) is both $p$ and phase dependent and is satisfied only if $p= \pm 1$ and $\delta \phi=0, \pi$. Substituting Eq. (11) into Eq. (9), with the addition that $\delta_{2}-\delta_{1}=\omega_{21}=\omega_{2}-\omega_{1}$ we find that, if Eq. (11) is satisfied, the zero root of the characteristic equation occurs when the lasers are tuned such that $\delta_{1}=-\Gamma_{1} \omega_{21} /\left(\Gamma_{1}+\Gamma_{2}\right)$ and $\delta_{2}=\Gamma_{2} \omega_{21} /\left(\Gamma_{1}+\Gamma_{2}\right)$.

This condition, Eq. (11), will lead to steady state population trapping in the system. If the system is initially in the ground state $\left[a_{0}(0)=1, a_{1}(0)=a_{2}(0)=0\right]$ then the asymptotic populations as $t \rightarrow \infty$ are given by $P_{0}=\omega_{21}^{4} /\left(\omega_{21}^{2}+8 \Omega^{2}\right)^{2}, \quad P_{1}=P_{2}=4 \Omega^{2} \omega_{21}^{2} /\left(\omega_{21}^{2}+\right.$ $\left.8 \Omega^{2}\right)^{2}$, when $\Omega_{01}^{a}=\Omega_{02}^{b}=\Omega, \Gamma_{1}=\Gamma_{2}$. Related results have been obtained with a single laser excitation [11]; however, in that case Eq. (11) is more restrictive since it does not depend on the laser intensity. In Fig. 2 we show the spontaneous emission spectrum of an atom initially in the ground state for four different phase values with atomic parameters that satisfy Eq. (11). The importance of the relative phase in the control of spontaneous emission is now obvious, as the spectrum is clearly double peaked for $\delta \phi=0$, but for $\delta \phi=\pi / 10$ a very narrow central peak appears. Increasing the phase difference $\delta \phi$, the spectrum becomes clearly triple peaked for $\delta \phi=\pi / 2$. However, for $\delta \phi=\pi$ the central peak is suppressed. The cancellation of the central peak for $\delta \phi=0$ is an effect of quantum interference [11]. However, in this case, by changing the phase difference $\delta \phi$, we can produce extreme spectral narrowing for phases around $\delta \phi=0$ and strong suppression of the central peak for $\delta \phi=\pi$. The extreme narrowing of the central peak, as observed in Fig. 2(b), occurs for parameters which slightly differ from those which satisfy the trapping condition (11). This is associated with the slow decay of
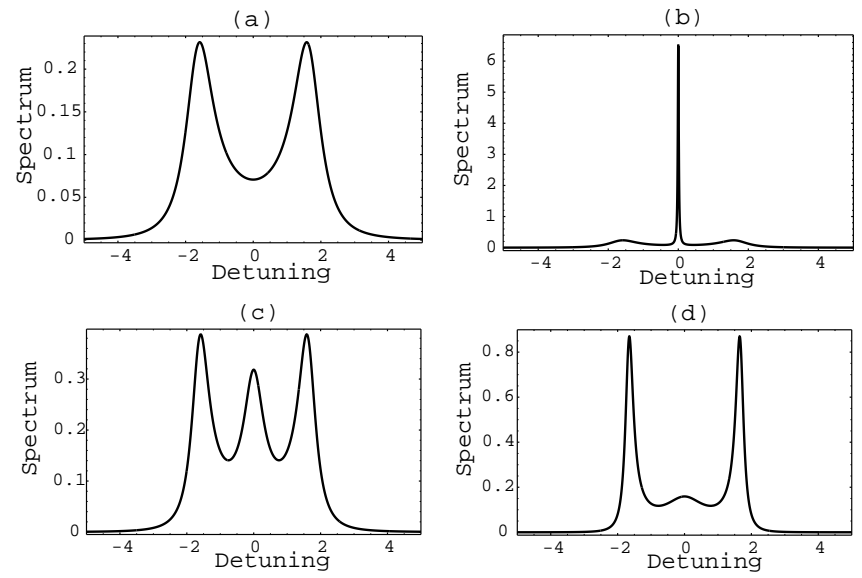

FIG. 2. The spontaneous emission spectra $S\left(\delta_{\mathbf{k}}\right)$ (in arbitrary units) for $\Omega_{10}^{a}=\Omega_{20}^{b}=\Gamma_{2}=\Gamma_{1}, \quad \Omega_{20}^{a}=\Omega_{10}^{b}=0, \omega_{21}=$ $2 \Gamma_{1}, \quad \delta_{1}=-\delta_{2}=-\Gamma_{1}$, and $p=1$. In (a) $\delta \phi=0$, (b) $\delta \phi=0.1 \pi$, (c) $\delta \phi=0.5 \pi$, and (d) $\delta \phi=\pi$. 
one of the dressed states of the atom, as analyzed by Zhou and Swain in the context of resonance fluorescence using a closed V-type atom [9]. In the dressed state picture the scheme of Fig. 1 can be seen as being three different dressed states decaying to state $|3\rangle$. The widths of these dressed states depend crucially on the relative phase $\delta \phi$. In particular, for Fig. 2(b) the width of the dressed state which is responsible for the central peak scales as $(\delta \phi)^{2}$ in the regime of $\delta \phi \approx 0$, a result that is obtained after making a Taylor expansion about $\delta \phi=0$, explaining the extreme narrowing observed.

In the above case the two excited states were well separated $\left(\omega_{21} \neq 0\right)$. In the case that the upper states are degenerate $\left(\omega_{21}=0\right)$ there is a second, new, condition for population trapping. This condition will produce two real roots for the characteristic equation of the Hamiltonian and lead the system to total population trapping, if the atom is initially in the ground state. In this case the atom will oscillate in a superposition of states $|0\rangle,|1\rangle,|2\rangle$ totally immune to any decay to state $|3\rangle$ due to total destructive quantum interference between the two transition paths $\left(|0\rangle \stackrel{\omega_{a}}{\rightarrow}|1\rangle \rightarrow|3\rangle\right)$ and $\left(|0\rangle^{\omega_{b}}|2\rangle \rightarrow|3\rangle\right)$. This condition is

$$
p \cos [\delta \phi]= \pm 1, \quad \sqrt{\Gamma_{1}} \Omega_{01}^{a}=\mp \sqrt{\Gamma_{2}} \Omega_{02}^{b},
$$

and can be satisfied by choosing the laser intensities such that $\sqrt{I_{a}} / \sqrt{I_{b}}=\mp \sqrt{\Gamma_{2}} A_{02} / \sqrt{\Gamma_{1}} A_{01}$. In Fig. 3 we present the fluorescence spectra for atomic parameters that satisfy Eq. (12) and the atom initially in the ground state. For these parameters, the spectrum is double peaked for $\delta \phi=0$, but for $\delta \phi=\pi / 2$ a zero value appears for $\delta_{\mathbf{k}}=0$. Furthermore, the system shows extreme linewidth narrowing for phases around $\pi$, and complete spontaneous emission cancellation for every vacuum mode due to total population trapping for $\delta \phi=$ $\pi$. In Fig. 3(c) the widths of the two dressed states responsible for the two side peaks are found to be the same and scale as $(\pi-\delta \phi)^{2}$ when $\delta \phi \approx \pi$ which explains the narrowing.

From Eqs. (5)-(8) with $p=1$ and the atom initially in the ground state we can easily obtain an analytical formula for $c_{\mathbf{k}}(t \rightarrow \infty)$. Then, in the nondegenerate case if $\delta \phi=0$ the spectrum has a zero value for $\delta_{\mathbf{k}}=$ $\left(\delta_{1} \sqrt{\Gamma_{2}} \Omega_{02}^{b}+\delta_{2} \sqrt{\Gamma_{1}} \Omega_{01}^{a}\right) /\left(\sqrt{\Gamma_{1}} \Omega_{01}^{a}+\sqrt{\Gamma_{2}} \Omega_{02}^{b}\right)$ and spontaneous emission is completely cancelled for this specific vacuum mode. An exception is the case when $\delta_{2}=-\delta_{1}=\omega_{21} / 2, \quad \Omega_{01}^{a}=\Omega_{02}^{b}$, and $\Gamma_{1}=\Gamma_{2}$, where the $\delta_{\mathbf{k}}$ term factors from both numerator and denominator and cancels [see Fig. 2(a)]. If now $\delta \phi=\pi$ the zero appears at $\delta_{\mathbf{k}}=$ $\left(\delta_{1} \sqrt{\Gamma_{2}} \Omega_{02}^{b}-\delta_{2} \sqrt{\Gamma_{1}} \Omega_{01}^{a}\right) /\left(\sqrt{\Gamma_{2}} \Omega_{02}^{b}-\sqrt{\Gamma_{1}} \Omega_{01}^{a}\right.$ ) (for $\left.\sqrt{\Gamma_{2}} \Omega_{02}^{b} \neq \sqrt{\Gamma_{1}} \Omega_{01}^{a}\right)$, with the exception when $\delta_{2}=-\delta_{1}=\omega_{21} / 2, \quad \Omega_{01}^{a}=-\Omega_{02}^{b}, \quad$ and $\quad \Gamma_{1}=\Gamma_{2}$. In the degenerate case $\delta_{1}=\delta_{2}=\delta$, a zero always appears in the spectrum at $\delta_{\mathbf{k}}=\delta$, independent of the values of the Rabi frequencies, decay rates, and relative phase. An example of this is Fig. 3(b) where the zero
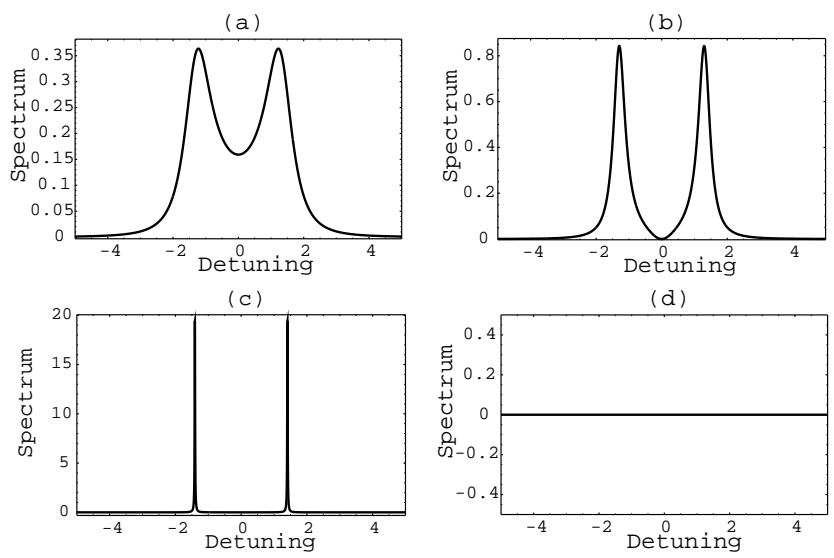

FIG. 3. The same as Fig. 2 but with $\delta_{1}=\delta_{2}=\omega_{21}=0$. In (a) $\delta \phi=0$, (b) $\delta \phi=0.5 \pi$, (c) $\delta \phi=0.9 \pi$, and (d) $\delta \phi=\pi$.

appears at $\delta_{\mathbf{k}}=0$. An exception is the case when $\Omega_{01}^{a}=\Omega_{02}^{b}$ and $\Gamma_{1}=\Gamma_{2}$ [see Fig. 3(a)].

Let us now suppose that each of the lasers can couple both of the excited states. Trapping conditions similar to Eqs. (11) and (12) can be derived but this will be discussed elsewhere. In Fig. 4 we show the phase dependence of the spontaneous emission spectrum for two values of $p \neq 1$, and the system initially in the ground state. The parameters chosen lead to steady state population trapping in the system if $\delta \phi=0$ and $p=1$ [extension of condition Eq. (11)]. The behavior of the atom is similar in both cases as the spectrum is triple peaked for $\delta \phi=0$ but as the phase increases towards $\pi$ the central peak dominates and the spectrum becomes single peaked. If a single laser is used for the excitation [11], for $p=0$ (orthogonal matrix elements) no cancellation of spontaneous emission is observed. However, in this case the two side peaks for $\delta \phi=0$ disappear towards $\delta \phi=\pi$. Obviously, in the case when $\Omega_{01}^{a}=\Omega_{01}^{b}, \Omega_{02}^{a}=\Omega_{02}^{b}$, and $\delta \phi=\pi$ there is no net field applied to the atom. Then, the atom will remain in the ground state if it is initially in the ground state or will behave as Agarwal [2] and others [3,20] described if it is initially in a superposition of the two excited states. Furthermore, in Fig. 5 we plot the spontaneous emission spectrum for arbitrary atomic parameters and $p=1$ for two different values of $\delta \phi$. The phase effect is also obvious here. The zeros in
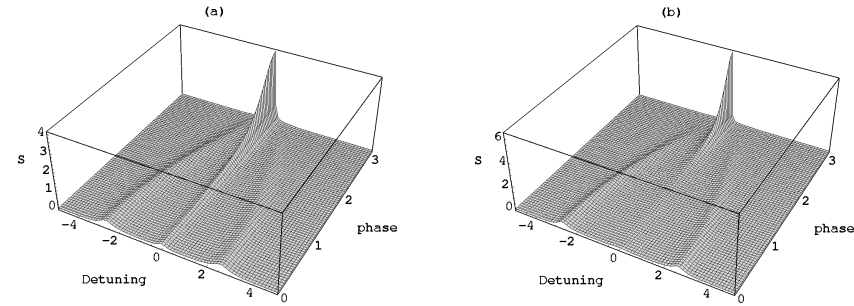

FIG. 4. $\quad S\left(\delta_{\mathbf{k}}\right)$ as a function of $\delta \phi$ for $\Omega_{10}^{a}=\Omega_{20}^{b}=\Gamma_{2}=$ $\Gamma_{1}, \Omega_{20}^{a}=\Omega_{10}^{b}=0.75 \Gamma_{1}, \omega_{21}=2 \Gamma_{1}$, and $\delta_{1}=-\delta_{2}=-\Gamma_{1}$. In (a) $p=0.5$ and (b) $p=0$. 

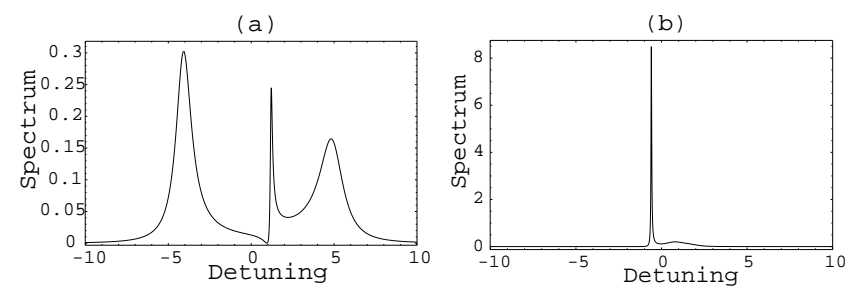

FIG. 5. The same as Fig. 5 for $\Gamma_{2}=2 \Gamma_{1}, \Omega_{10}^{a}=1.5 \Gamma_{1}$, $\Omega_{20}^{b}=\Gamma_{1}, \Omega_{20}^{a}=1.75 \Gamma_{1}, \Omega_{10}^{b}=2 \Gamma_{1}, \quad \omega_{21}=2 \Gamma_{1}, \quad \delta_{1}=0$, $\delta_{2}=2 \Gamma_{1}$, and $p=1$. In (a) $\delta \phi=0$ and (b) $\delta \phi=\pi$.

the spectra appear at values of similar form to those we have predicted above: e.g., if $\delta \phi=0$ the zero appears at $\delta_{\mathbf{k}}=\left[\delta_{1} \sqrt{\Gamma_{2}}\left(\Omega_{02}^{a}+\Omega_{02}^{b}\right)+\delta_{2} \sqrt{\Gamma_{1}}\left(\Omega_{01}^{a}+\right.\right.$ $\left.\left.\Omega_{01}^{b}\right)\right] /\left[\sqrt{\Gamma_{1}}\left(\Omega_{01}^{a}+\Omega_{01}^{b}\right)+\sqrt{\Gamma_{2}}\left(\Omega_{02}^{a}+\Omega_{02}^{b}\right)\right], \quad$ as shown in Fig. 5(a).

In summary, we have demonstrated that spontaneous emission from an open V-type atom can be controlled via the phase difference of the two lasers used for the excitation. Effects such as partial cancellation, extreme linewidth narrowing, and complete cancellation of the fluorescence of such an atom have been predicted, even in nontrapping conditions. For an experimental realization of this proposal, the successful experiment of Xia et al. [12] in sodium dimers should be modified only by the addition of another laser which will couple the system with a four-photon transition. The phase of the two commensurate frequencies can be varied using standard phase control techniques [13]. We should also note that, as Fig. 5 indicates, this scheme is quite robust and large modification of spontaneous emission can be achieved even in the case that the atomic and laser parameters do not satisfy any of the population trapping conditions.

This work was funded in part by the U.K. Engineering and Physical Sciences Research Council and the European Community.

[1] G. Alzetta et al., Nuovo Cimento Soc. Ital. Fis. 36B, 5 (1974); E. Arimondo, in Progress in Optics, edited by E. Wolf (Elsevier, Amsterdam, 1996), Vol. XXXV, p. 257.

[2] G.S. Agarwal, Quantum Optics (Springer-Verlag, Berlin, 1974).
[3] H. R. Gray et al., Opt. Lett. 6, 218 (1978); P. L. Knight, J. Phys. B 12, 3297 (1979); D. A. Cardimona et al., J. Phys. B 15, 55 (1982); D. Agassi, Phys. Rev. A 30, 2449 (1984).

[4] O. Kocharovskaya and Ya. I. Khanin, JETP Lett. 48, 630 (1988); S. E. Harris, Phys. Rev. Lett. 62, 1033 (1989); M. O. Scully et al., ibid. 62, 2813 (1989); A. Imamoḡu, Phys. Rev. A 40, 2835 (1989); S. E. Harris and J. J. Macklin, ibid. 40, 4135 (1989); G. S. Agarwal, Phys. Rev. Lett. 67, 980 (1991); M. Fleischhauer et al., Opt. Commun. 94, 599 (1992); J. Javanainen, Europhys. Lett. 17, 407 (1992); C. H. Keitel et al., Phys. Rev. A 48, 3196 (1993).

[5] L. M. Narducci et al., Phys. Rev. A 42, 1630 (1990); D. J. Gauthier et al., Phys. Rev. Lett. 66, 2460 (1991); C. H. Keitel et al., Appl. Phys. B 60, S153 (1995).

[6] G. C. Hegerfeldt and M. B. Plenio, Phys. Rev. A 46, 373 (1992); 47, 2186 (1993).

[7] S. Schiemann et al., Phys. Rev. Lett. 71, 3637 (1993); J. Martin et al., Phys. Rev. A 54, 1556 (1996).

[8] S.-Y. Zhu et al., Phys. Rev. A 52, 4791 (1995).

[9] P. Zhou and S. Swain, Phys. Rev. Lett. 77, 3995 (1996); Phys. Rev. A 56, 3011 (1997).

[10] G. S. Agarwal, Phys. Rev. A 54, R3734 (1996); 55, 2457 (1997).

[11] S.-Y. Zhu and M.O. Scully, Phys. Rev. Lett. 76, 388 (1996); H. Huang et al., Phys. Rev. A 55, 744 (1997); H. Lee et al., ibid. 55, 4454 (1997).

[12] H.-R. Xia et al., Phys. Rev. Lett. 77, 1032 (1996).

[13] C. Chen and D. S. Elliott, Phys. Rev. Lett. 65, 1737 (1990); L. Zhu et al., Science 270, 77 (1995).

[14] M. Shapiro et al., Chem. Phys. Lett. 149, 451 (1988); T. Nakajima and P. Lambropoulos, Phys. Rev. Lett. 70, 1081 (1993); M. Protopapas and P. L. Knight, J. Phys. B 28, 4459 (1995).

[15] M. A. G. Martinez et al., Phys. Rev. A 55, 4483 (1997).

[16] T. Quang et al., Phys. Rev. Lett. 79, 5238 (1997).

[17] Q. Wu et al., Phys. Rev. A 49, R1519 (1994); 50, 1474 (1994).

[18] Z. Ficek and H.S. Freedhoff, Phys. Rev. A 48, 3092 (1993); H.S. Freedhoff and Z. Ficek, ibid. 55, 1234 (1997).

[19] S. M. Barnett and P.M. Radmore, Methods in Theoretical Quantum Optics (Oxford University Press, Oxford, 1997).

[20] S.-Y. Zhu et al., Phys. Rev. A 52, 710 (1995); P. Zhou and S. Swain, Phys. Rev. Lett. 78, 832 (1997). 